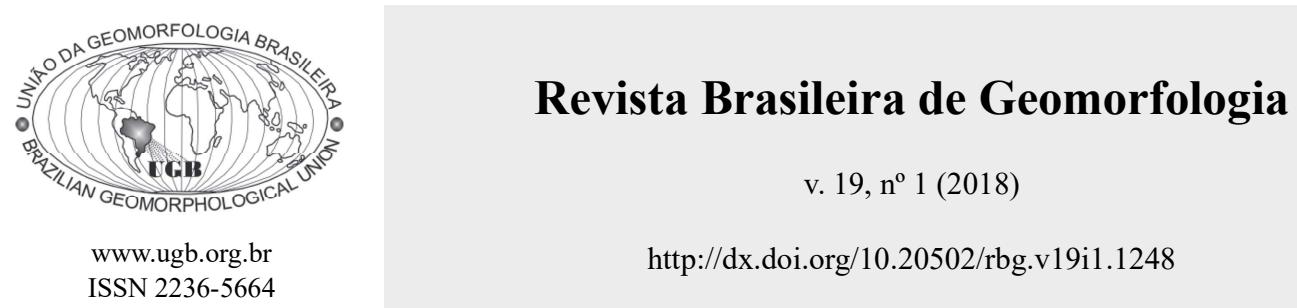

\title{
DINÂMICA DO CARBONO ORGÂNICO E PROCESSOS HIDROLÓGICOS NA ESCALA DA BACIA HIDROGRÁFICA: UMA REVISÃO
}

\section{ORGANIC CARBON DYNAMIC AND HYDROLOGICAL PROCESSES ON CATCHMENT SCALE: A REVIEW}

\author{
Cesar Augusto Crovador Siefert \\ Departamento de Geografia, Universidade Federal do Paraná, Centro Politécnico \\ Curitiba, Paraná, CEP 81531-990, Brasil \\ Email: cesarsiefert@ufpr.br \\ Irani dos Santos \\ Departamento de Geografia, Universidade Federal do Paraná Centro Politécnico \\ Curitiba, Paraná, CEP: 81531-990, Brasil \\ Email:irani@ufpr.br
}

\section{Informações sobre o Artigo \\ Recebido (Received): \\ 30/03/2017 \\ Aceito (Accepted): \\ $10 / 08 / 2017$}

\section{Palavras-chave:}

Ciclo do Carbono; Vertentes;

Biogeoquímica.

\section{Keywords:}

Carbon Cycle; Hillslopes;

Biogeochemistry.

\section{Resumo:}

Os processos de armazenamento e transferência de carbono orgânico (CO) em bacias hidrográficas são governados por interações entre processos hidrológicos e biogeoquímicos. Estudos clássicos evidenciam o papel da matéria orgânica na estabilização dos agregados do solo, propriedades físico-químicas e qualidade do solo, entretanto os mecanismos de desestabilização do carbono orgânico, armazenamento e transporte ainda são parcialmente descritos e compreendidos. Este artigo tem como objetivo apresentar uma revisão conceitual sobre a dinâmica do carbono orgânico enfatizando as interações entre os processos hidrológicos, sedimentológicos e biogeoquímicos na escala da bacia hidrográfica. $\mathrm{O}$ funcionamento da bacia hidrográfica, a partir das interações ocorridas entre zonas ripárias, vertentes e canal, refletem a variabilidade temporal e heterogeneidade espacial da concentração de carbono orgânico dissolvido (DOC) e carbono orgânico particulado (POC) no canal e aquífero raso. Desta maneira, descreve-se aqui os principais mecanismos responsáveis pelas interações entre solo e água e o ciclo do carbono orgânico na paisagem. Além disso, busca-se evidenciar possíveis incertezas e lacunas na descrição e compreensão dos processos relacionados a dinâmica do carbono orgânico em bacias hidrográficas.

\footnotetext{
Abstract:

Storage and fluxes of organic carbon (OC) on catchments are governed by interactions between hydrological and biogeochemical processes. Classical studies show the role of organic matter on the aggregate stability, physical and chemical properties and soil quality. However, the main mechanisms of organic carbon destabilization, storage and fluxes remains partially described and understood.
} 
This paper presents a conceptual review describing the organic carbon dynamic emphasizing the interactions between hydrological, sedimentological and biogeochemical processes on catchment scale. Catchment functioning through the interactions of riparian zones, hillslopes and streams reflects the temporal variability and the spatial heterogeneity of dissolved (DOC) and particulate (POC) organic carbon on streams and shallow groundwater. Thus, here we described the role of the main mechanisms responsible for interactions between soil and water and the organic carbon cycle on landscape. Furthermore, we seek to evidence uncertainties and gaps on describing and understanding of the processes related with the organic carbon dynamic on catchments.

\section{Introdução}

É amplamente reconhecido que o transporte de compostos orgânicos pela água em ambientes florestais estabelece um importante meio de ligação entre os ecossistemas aquáticos e terrestres (Dosskey $\&$ Bertsch, 1994). Estudos clássicos evidenciam o papel da matéria orgânica na estabilização dos agregados do solo, atributos físico-químicos e qualidade do solo (p.ex. Tisdall \& Oades, 1982; Doran \& Parkin, 1994), entretanto os mecanismos de desestabilização de carbono orgânico ainda são parcialmente descritos e compreendidos (Schmidt et al., 2011). Enquanto as emissões de formas gasosas de carbono receberam grande interesse da comunidade científica nos últimos anos devido a relação com aquecimento global e efeito estufa (Lal, 2004), ainda existem diversas lacunas na descrição das perdas de matéria orgânica na fração dissolvida e particulada e sua relação com processos hidrológicos e sedimentológicos.

A concentração de carbono orgânico, seja nas frações particulada (POC) ou dissolvida (DOC), em canais fluviaisé um dos elementos chaves na compreensão do ciclo global do carbono, sobretudo na interação entre os ambientes terrestres e aquáticos a partir de processos hidrológicos, sedimentológicos e biogeoquímicos relacionados. Entretanto algumas incertezas ainda permanecem quanto as suas fontes, dinâmicas e aporte na rede de drenagem em pequenas bacias (Chaplot \& Ribolzi, 2014), onde processos em vertentes são determinantes. Em pequenas bacias hidrográficas (menores que $5 \mathrm{~km}^{2}$ ) como a heterogeneidade espacial de processos hidrogeomorfológicos e parâmetros geomorfométricas é acentuada, são observadas fortes correlações entre diversas variáveis e a dinâmica de CO no canal (Hope et al., 1997; Dawson et al., 2004; Birkel et al., 2014; Dick et al., 2015).

Neste contexto, compreender a dinâmica do carbono orgânico $(\mathrm{CO})$ em bacias hidrográficas torna-se essencial para elucidar os mecanismos de armazenamento e transporte por três razões principais: (i) grande quantidade de $\mathrm{CO}$ exportado dos solos para os canais ocorrem durante os eventos pluviométricos; (ii) variações da concentração de $\mathrm{CO}$ em frações dissolvidas e particuladas em pequena escala temporal podem gerar aproximações de como o CO é transferido pela paisagem até os canais e (iii) determinar os padrões espaciais das áreas fontes e relacionar a dinâmica de DOC e POC aos mecanismos de geração de escoamento em bacias.

Desta maneira, este artigo apresenta uma revisão bibliográfica da dinâmica de carbono orgânico em bacias hidrográficas a partir dos seguintes objetivos: (i) apresentar revisão conceitual sobre a dinâmica do carbono orgânico enfatizando as interações entre os processos hidrológicos, sedimentológicos e biogeoquímicos na escala da bacia hidrográfica com ênfase nos processos de vertentes; (ii) identificação de eventuais lacunas observadas na compreensão dos processos e oportunidades de avanços na descrição da relação entre processos hidrológicos, sedimentológicos e biogeoquímicos associados ao ciclo do carbono orgânico na escala da bacia hidrográfica.

\section{Ciclo Global do Carbono}

Em escala global o carbono circula por 5 reservatórios (pools) principais: aquático, atmosférico, geológico, solo e biota, considerando um estoque aproximado de $46.820 \mathrm{Gt}$, distribuídos na paisagem. Além destes, existe ainda um volume de aproximadamente 8,3 Gt estocados juntamente com os combustíveis fósseis (carvão mineral, petróleo, gás natural e peatlands) (Falkowski et al., 2000).

De forma simplificada, a representação do ciclo global do carbono envolve apenas 3 reservatórios: sendo 2 deles biologicamente ativos, oceânico e terrestre (solo e vegetação), conectados a atmosfera via fluxos gasosos (Falkowski et al., 2000). Todos os reservatórios são 
interconectados, ocorrendo a circulação do carbono entre eles (Lal, 2004) mediante a ocorrência de processos de origem hidro-bio-geoquímicos. Desta forma, as alterações na concentração de carbono nos diferentes pools dependem, não apenas das atividades humanas, mas também dos processos biogeoquímicos e climáticos e suas interações com o ciclo do carbono.

Considerando apenas os compartimentos terrestres (solo, biota e atmosfera), o solo possui o maior estoque disponível de carbono, sobretudo ao considerar que dos 2.500 Gt disponíveis no solo, aproximadamente 1.550 Gt são referentes ao carbono orgânico (CO), estocado sob a forma de matéria orgânica (Lal, 2004). A concentração de CO é extremamente variável em solos de acordo com as condições climáticas, sendo baixa em solos de regiões áridas, alto em zonas temperadas e extremamente alto em solos de origem orgânica em zonas tropicais (Lal, 2004).

Embora a transferência de carbono mediante fluxos entre as superfícies continentais e a atmosfera sejam superiores aos mecanismos associados as águas superficiais (Lafleur et al., 2003; Billet et al., 2006), as taxas de aporte e transporte de DOC em canais inseridos em bacias hidrográficas com a presença de solos orgânicos podem ser comparáveis as taxas de sequestro de carbono no contexto solo - planta (Hope et al., 2004). Cole et al. (2007) afirmam ainda que a transferência de $\mathrm{C}$ terrestre para as águas interiores é maior que o volume de carbono que é exportado para os oceanos e aproximadamente similar a produção líquida do ecossistema (NEP, do inglês Net Ecossystem Production).

$\mathrm{Na}$ escala da bacia hidrográfica, o carbono é armazenado em diversos pools (p.ex: biomassa da parte aérea da vegetação, raízes, solo, vegetação herbácea e liteira) de tamanhos e distribuição variáveis de acordo com as características climáticas, manejo do solo, perturbação e nível de degradação (Eglin et al., 2008). Em geral, as estimativas referentes as interações de $\mathrm{CO}$ em escala global são realizadas indiretamente e tendem a gerar incertezas quanto a quantificação em alguns reservatórios devido as simplificações adotadas (Cole et al., 2007). Assim, considerando a necessidade de compreender detalhadamente o ciclo do carbono, diversas lacunas ainda existem, por exemplo: (i) quantificação dos processos erosivos envolvendo o transporte de carbono orgânico, (ii) na distribuição espacial pela paisagem e (iii) na quantificação do aporte de carbono orgânico nos sistemas fluviais (Chaplot et al., 2005), sendo ainda evidenciada incertezas na descrição e modelagem dos processos de transporte de DOC via processos hidrológicos superficiais e subsuperficiais (Mei et al., 2012).

\section{Dinâmica do Carbono Orgânico entre Processos Hidrológicos e Biogeoquímicos na Escala da Bacia Hidrográfica}

\section{Ciclo do Carbono Orgânico em bacias hidrográficas}

O ciclo do carbono pode ser conceitualmente descrito a partir de três compartimentos de origem (i) terrestre (solo e vegetação), (ii) atmosférica e (iii) oceânica, sendo que o ecossistema terrestre abarca os processos físicos e químicos oriundos da interação entre as características biogeoquímicas entre solos, vegetação e água na escala da bacia hidrográfica (Figura 1).

Neste sentido, características de origem climática, geológica, associadas a práticas de uso e manejo em distintas unidades pedológicas e vegetação tem sido identificadas como os principais fatores que atuam nos controles iniciais do volume de carbono estocado no compartimento terrestre (Yoo et al., 2006; Dawson \& Smith, 2007). Conforme Lal (2004), o estoque global de carbono orgânico no solo na camada de profundidade de 0 - $1 \mathrm{~m}$ é de cerca de 1.550 Gt (Jobbágy \& Jackson, 2000), armazenado em forma de matéria orgânica, cerca de 3 vezes superior ao estoque na atmosfera (Davidson \& Janssens, 2006) e 4,5 vezes superior ao estoque na biota, estando em equilíbrio dinâmico com o ambiente (Lal, 2005).

Compreender os padrões e processos envolvidos na redistribuição do SOC (Soil Organic Carbon) associado ao ciclo hidrossedimentológico em ambientes florestais e agrícolas torna-se elemento chave para estimar o potencial de sequestro de carbono nestes sistemas, bem como fornecer subsídios para ao desenvolvimento de modelos que possam predizer o padrão espacial e os mecanismos de transferência de CO na paisagem (Ritchie et al., 2007). Além disso, reconhece-se que as planícies de inundação atuam como importantes hotspots devido ao armazenamento de carbono orgânico na paisagem em função da deposição de sedimentos aluviais enriquecidos (Hoffmann et al., 2009) e de frações de CO oriundos de montante (Ritchie 
et al., 2007), tornando evidente relação entre topografia e o padrão espacial das fontes de carbono orgânico em bacias hidrográficas permitindo a sua aproximação e estimativa mediante uso de atributos geomorfométricos da paisagem (Schwanghart \& Jarmer, 2011). E, de maneira geral, as concentrações de SOC decrescem na medida em se aumentam os gradientes de declividade e vertentes côncavas possuem maiores concentrações de $\mathrm{CO}$ no perfil do solo do que vertentes convexas (Ritchie et al., 2007).

Os processos erosivos atuam como principais agentes no transporte e deposição superficial do carbono orgânico na paisagem (Starr et al., 2006; Ritchie et al., 2007; Schwanghart \& Jarmer, 2011), promovendo ainda a remoção seletiva dos agregados do solo (Thomaz \& Vestena, 2012). Considerando a desagregação primária de partículas mais finas (diâmetros reduzidos) e mais leves (de menor densidade) (Lal, 1995), os sedimentos são usualmente enriquecidos não apenas com as partículas de silte e argila transportadas em conjunto com as formas estáveis de carbono orgânico presentes no solo (Baldock \& Skjemstad, 2000), mas também de matéria orgânica particulada de fácil decomposição (Chaplot et al., 2005).

O ciclo do carbono em ambiente terrestres é intimamente associado ao ciclo hidrológico (Figura 2) e considera-se ainda que os processos hidrológicos atuem como mecanismos de controle primário na transferência e redistribuição de matéria orgânica e nutrientes na paisagem, sobretudo o carbono orgânico nas frações dissolvidas e adsorvidas (Boyer et al., 1996; Hinton et al., 1998; Frank et al., 2000; McGlynn \& McDonnell, 2003a; Dalzell et al., 2007; Temnerud et al., 2007; Lambert et al., 2011; Lambert et al., 2013), sugerindo que a variabilidade inerente dos processos hidrológicos que irá produzir diferentes padrões temporais e espaciais na dinâmica dos nutrientes nas vertentes, zona ripária e canal (Stieglitz et al., 2003; van Verseveld et al., 2009).

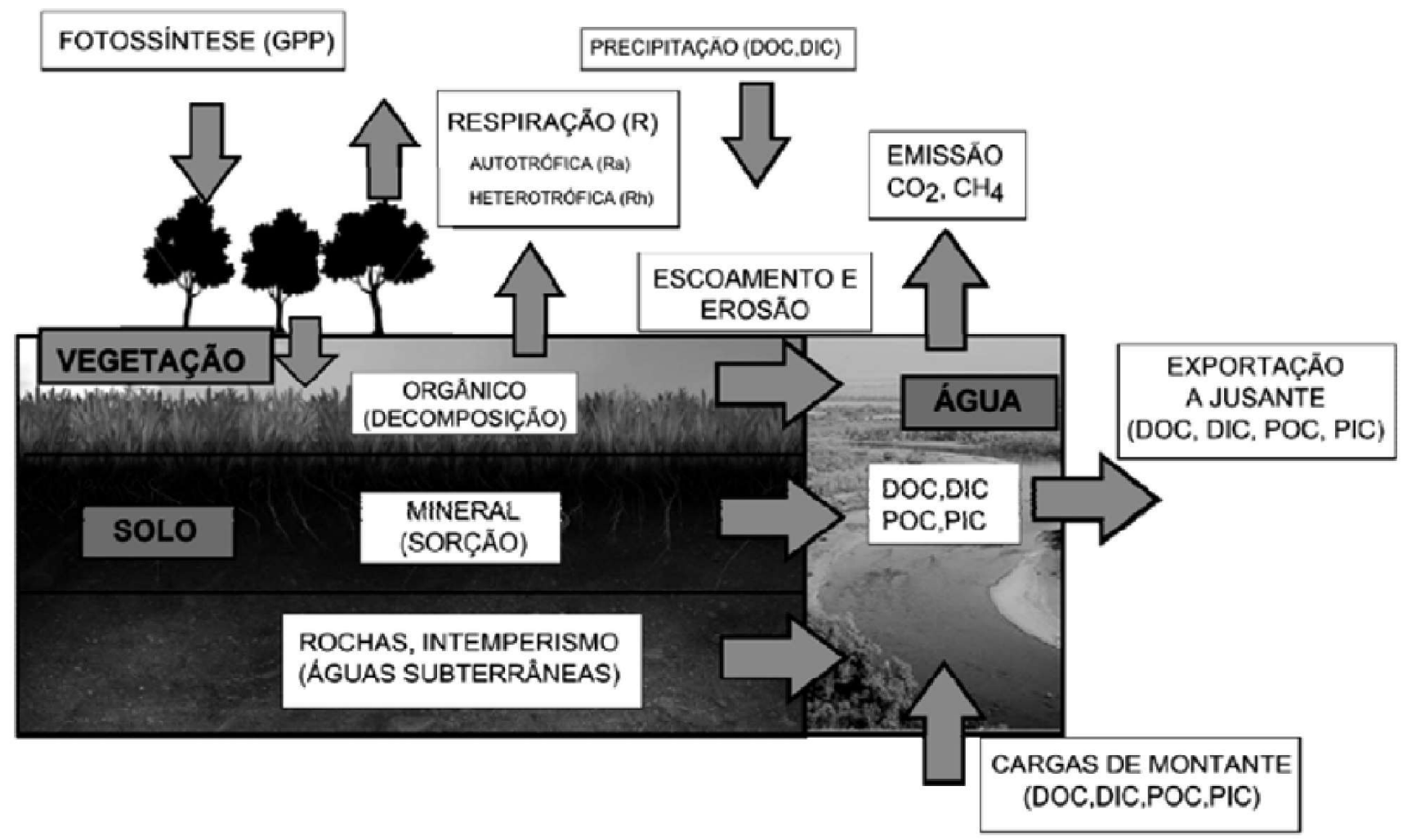

Figura 1 - Vias de fluxo de Carbono no ecossistema terrestre.

onde: Carbono Orgânico Dissolvido (DOC), Carbono Orgânico Particulado (POC), Carbono Inorgânico Dissolvido (DIC), Carbono Inorgânico Particulado (PIC).

Fonte: Sorribas (2011). 
O CO é fonte de energia para os ecossistemas aquáticos e, quimicamente, ainda participa dos mecanismos de transporte e disponibilidade de íons, nutrientes, metais pesados e poluentes orgânicos (Boyer et al., 1996). E, do ponto de vista biogeoquímico, o fato da concentração e da caracterização química do carbono orgânico total (TOC, Total Organic Carbon) ser variável, denota ainda que as frações dissolvidas (DOC) e particulada (POC) são variáveis durante eventos pluviométricos, indicando que a contribuição e aporte no canal é oriunda de diversas áreas fonte (Hood et al., 2006).

As concentrações de DOC e POC em canais remetem as suas origens em ambientes pedológicos, dado o estoque de carbono e percentual de solos orgânicos na bacia hidrográfica (Aitkenhead et al., 1999; Dawson et al., 2004). Ao estimar os fluxos de exportação de carbono em 175 rios em ambiente tropical, Huang et al., (2012) indicaram que a soma DOC + POC correspondem, no mínimo, a $50 \%$ do aporte total de carbono nos oceanos, enfatizando ainda que os rios do continente americano possuem os maiores aportes de DOC em escala global. Em longo prazo, as concentrações de DOC e POC apresentam comportamento distinto no canal, por exemplo, em climas temperados enquanto o DOC apresenta um padrão sazonal, com uma elevada concentração durante o verão e picos durante o outono cuja tendência é alterada em virtude de eventos pluviométricos, o POC apresenta uma dinâmica relacionada somente a eventos e maiores concentrações em virtude dos picos de vazão devido ao incremento do aporte de sedimentos no canal via escoamento superficial ao promover a remoção e transporte dos agregados do solo (Dawson et al., 2004). Aitkenhead et al. (1999), ao investigarem a relação espacial entre a concentração de DOC no canal e os reservatórios de carbono orgânico no solo, observaram que as maiores correlações estatísticas entre estas duas variáveis são observadas em bacias hidrográficas com área de drenagem inferior a $5 \mathrm{~km}^{2}$.

Especificamente para rios brasileiros, Sorribas et al., (2012) obtiveram concentrações média de DOC de $1,38 \mathrm{mg} / \mathrm{L}$ e $1,16 \mathrm{mg} / \mathrm{L}$ para duas bacias hidrográficas $\left(84 \mathrm{~km}^{2}\right.$ e $19 \mathrm{~km}^{2}$, respectivamente) no sul do Brasil. Knapik (2014) obteve a variação espacial das concentrações de DOC em 6 pontos ao longo do

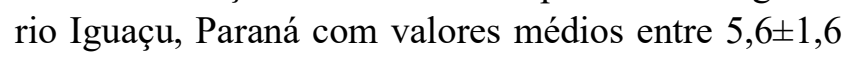
$\left(321,4 \mathrm{~km}^{2}\right)$ a $7,8 \pm 3,4 \mathrm{mg} / \mathrm{L}\left(666,2 \mathrm{~km}^{2}\right)$.
Por outro lado, em uma bacia de cabeceira recoberta com reflorestamento localizada na região sul brasileira, Siefert (2016) obteve concentrações médias de DOC de $2,46 \pm 0,8 \mathrm{mg} / \mathrm{L}$, inferior ao obtido no aquífero raso na zona ripária e vertentes da bacia. Analisando a relação entre TOC (DOC + POC) em uma bacia hidrográfica agrícola $\left(30 \mathrm{~km}^{2}\right)$ localizada na região sul-brasileira, Zucco et al., (2015) observou diferença significativa entre a ascensão e a recessão (menores concentrações de DOC) do hidrograma, indicando que os processos de transporte e mobilização de frações de carbono via processos de escoamento superficial atuantes nas vertentes relacionam-se diretamente com as variações de carbono nos canais. Na região amazônica brasileira, Waterloo et al., (2006) indicam que a exportação fluvial de carbono orgânico em uma bacia de cabeceira $\left(6,8 \mathrm{~km}^{2}\right)$ é essencialmente dependente do regime pluviométrico apresentando maiores concentrações de DOC (92 - 94\% da exportação total) em função dos picos do hidrograma e, por consequência, apresenta um forte indício de sazonalidade interanual. Entretanto, as concentrações de DOC no canal são superiores ao obtido na precipitação, indicando a produção e mobilização de DOC na bacia e aporte nos canais via processos de origem hidrológica e sedimentológica ocorridos nas vertentes (Waterloo et al., 2006).

No contexto da bacia hidrográfica, a concentração de DOC em canais é oriunda do ambiente terrestre como resultado das transformações químicas do carbono do solo como carbono orgânico sólido a partir da decomposição de materiais húmicos (SOC), emissão de $\mathrm{CO}_{2}$, DOC e carbono inorgânico dissolvido (DIC) (Lennon, 2004; Birkel et al., 2014).

Diversos experimentos evidenciam que cerca de $30 \%$ a $50 \%$ do volume total anual de DOC exportado pelos canais é devido a rápidas alterações em eventos pluviométricos com pequena duração e grandes intensidades que ocupam de $4 \%$ a $24 \%$ do tempo. Por outro lado, em alguns casos, cerca de $30 \%$ a $60 \%$ da carga anual de DOC exportada é oriunda de apenas um evento pluviométrico (Clark et al., 2007). Sendo assim, estima-se que em geral, o DOC em pequenos canais é oriundo de lixiviação de solos (dessorção SOC - DOC) e plantas, enquanto que o POC possui origem autóctone sendo sua concentração relacionada com a erosão do solo em ambientes sedimentares (Swarzenski \& Campbell 2005). 


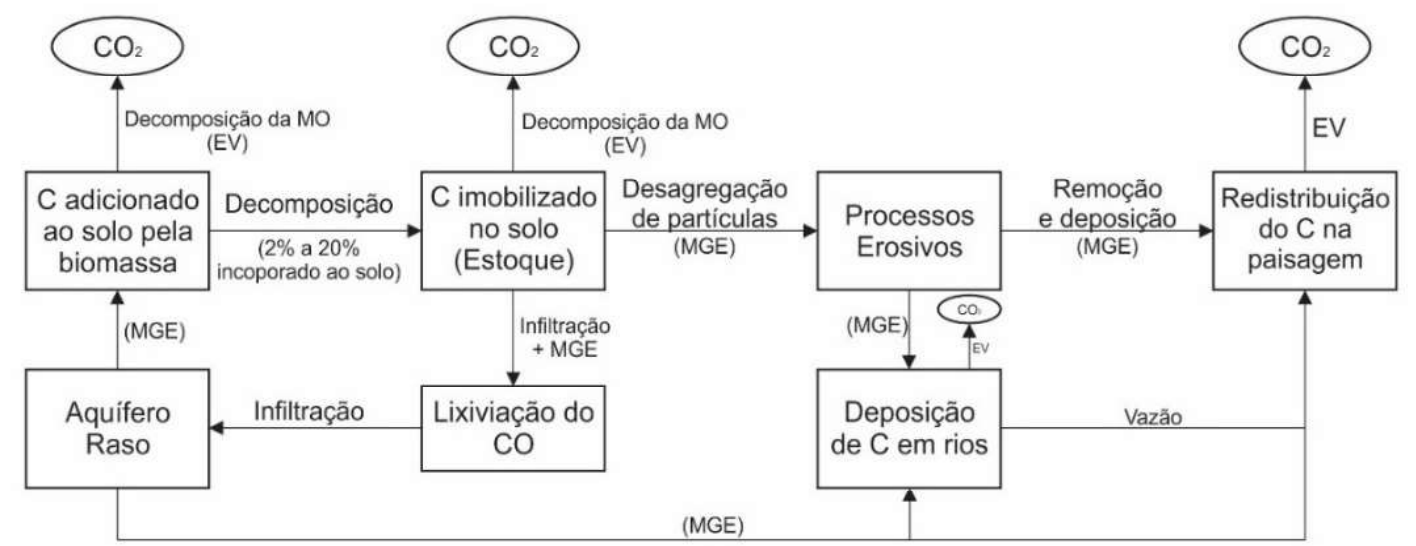

Figura 2 - Ciclagem do carbono orgânico em bacias hidrográficas e processos hidrológicos atuantes.

Fonte: adaptado de Lal (2004).

Em geral, os processos que dão origem a matéria orgânica (DOC e POC) na interação solo - água apresentam pequenas variações nas regiões tropicais, temperadas e boreais e são dependentes de fatores como: propriedades físicas do ambiente (temperatura do ar e solo e umidade), químicas (disponibilidade de nutrientes, concentração de oxigênio e reações de óxido-redução) e disponibilidade de microrganismos (Knapik, 2014). Com relação ao transporte de carbono essencialmente nos canais, Dawson et al. (2004), ao analisar três pontos de amostragem no canal principal de uma bacia hidrográfica $\left(1,3 \mathrm{~km}^{2}\right)$ dominada por solos orgânicos e embutida no rio Dee (Reino Unido), afirmam que o transporte de carbono orgânico é governado, primariamente, por processos abióticos e físicos (desgaseificação, deposição e resuspensão das partículas) e das condições de mistura via processos hidrológicos. Meybeck (1982) indica que as concentrações médias de DOC nas águas superficiais relacionam-se com as condições climáticas, onde regiões de clima temperado apresentam os maiores valores médios $(10 \mathrm{mg} / \mathrm{L})$ se comparado com regiões tropicais úmidas $(6 \mathrm{mg} / \mathrm{L})$.

Em média, os canais transportam uma carga de carbono orgânico dissolvido 6 a 10 vezes superior que a carga particulada (Thurman, 1985). A variação na concentração de DOC no canal pode ser explicada ainda pela relação sinérgica de diversos fatores, dentre eles as unidades pedológicas que ditarão as taxas de produção de DOC em solos orgânicos, adsorção de DOC em solos minerais (Aitkenhead et al., 1999) e os caminhos preferenciais de escoamento através dos diferentes horizontes do solo (Hinton et al., 1998; Lambert et al., 2011; Lambert et al., 2013). Desta maneira, espera-se que a extensão, localização espacial e conectividade dinâmica das áreas saturadas nas vertentes e zonas ripárias irão influenciar os padrões temporais de concentração de DOC e POC em canais (Inamdar \& Mitchell, 2006).

Birkel et al. (2014) ao descrever os processos de produção de DOC na bacia considera que a produção é descrita como sendo dependente da temperatura do ar e da umidade do solo, além da produção específica e armazenamento de DOC na fração de água estocada nas vertentes e zona ripária. Siefert (2016) indica que a produção de DOC em uma bacia de primeira ordem em região subtropical é essencialmente dependente das condições antecedentes de umidade e do nível do aquífero raso, uma vez que porções da bacia cujo nível do aquífero raso possui maior permanência próxima a superfície apresentam, por consequência, as maiores concentrações de DOC na fração de água armazenada nas encostas e posteriormente transferida para o canal. Marques et al., (2012) indicam que as concentrações médias de DOC na solução do solo em 3 parcelas experimentais sob diferentes usos do solo na região Amazônica apresentam variações temporal, espacial e em profundidade, indicando 3 zonas distintas de transporte de DOC no perfil do solo: superficial (0$30 \mathrm{~cm})$, intermediária $(40-100 \mathrm{~cm})$ e subsuperficial $(200 \mathrm{~cm})$.

A variabilidade temporal e espacial dos padrões de umidade do solo são elementos-chave para compreensão da contribuição das áreas fontes de escoamento, 
nutrientes e sedimentos nos canais (Geneviève et al., 2014). E, desta forma, um dos principais fatores físicos que irá influenciar a dinâmica de nutrientes e matéria orgânica no perfil do solo é a quantidade de precipitação associada a condições antecedentes de umidade (Stieglitz et al., 2003).

Neste sentido, enquanto a água promove o efeito de lixiviação dos nutrientes (movimento vertical e horizontal) e alteração nos padrões de umidade do solo, que afetará o volume de oxigênio no perfil do solo e a degradação microbiana, os caminhos preferenciais de escoamento e a distribuição espacial da água residual no perfil do solo na bacia também serão importantes para a elucidação do transporte de nutrientes como solutos (Frank et al., 2000). De maneira geral, van Verseveld et al. (2008) categorizam as explicações para o transporte subsuperficial de nutrientes e consequente aporte em canais em três abordagens principais: (i) ascensão do nível freático as camadas superficiais do solo com elevadas concentrações de nutrientes, (ii) transporte vertical de nutrientes via caminhos preferenciais no solo para a interface solo - manto rochoso com consequente escoamento lateral no sentido de jusante e (iii) transporte vertical de nutrientes e lateral no perfil do solo. Assim, torna-se evidente a relação entre a heterogeneidade espacial (vertical e horizontal) do SOC em solos e a variabilidade temporal de DOC e POC nos canais (Lambert et al., 2011), sendo que em geral, assume-se que durante eventos pluviométricos o suprimento de nutrientes (p.ex.: DOC e DON) não seja finito (McGlynn \& McDonnell, 2003a; Bishop et al., 2004).

O primeiro estágio de perda de carbono orgânico do solo para águas superficiais é o movimento de matéria orgânica, e consequentemente de DOC pela água retida no perfil do solo. Neste sentido, o material orgânico que compõe a serapilheira torna-se uma importante fonte de DOC em bacias hidrográficas florestadas a partir da infiltração e percolação da água passando por compostos orgânicos com elevadas taxas de concentração de $\mathrm{CO}$ adicionados ao solo (Hongve, 1999). Já o transporte lateral de solutos no perfil do solo ocorre pelos caminhos preferenciais de escoamento nas vertentes e zonas ripárias, sendo que esta fração da água se torna enriquecida pela movimentação da água nos horizontes minerais e/ou orgânicos em direção ao aquífero e canais (Dawson \& Smith, 2007).
Mecanismos controladores da variação espacial e temporal de CO na paisagem: interações entre zonas ripárias, vertentes e canal

Considerando a ideia de que a heterogeneidade espacial da paisagem é um dos mecanismos que controlam a concentração de DOC na rede de drenagem, Laudon et al. (2011) apresentaram um modelo simplificado para estimar a dinâmica de DOC no canal a partir da proporção de unidades de paisagem relacionadas ao uso do solo (i.e. florestas e peatlands) e sua contribuição relativa ao hidrograma. Denota-se que a variabilidade temporal da concentração de DOC no canal está relacionada a conectividade estabelecida entre os solos de cada unidade da paisagem e a rede de canais, sendo temporalmente dinâmica e susceptível a perturbações de natureza diversa.

Dada a distribuição não uniforme da concentração de carbono orgânico no perfil do solo, via de regra diminuindo em profundidade, sobretudo nas porções que apresentam saturação dinâmica do solo nas vertentes (Siefert, 2016), é esperado que o volume de água contida nos horizontes mais superficiais apresente elevadas concentrações de DOC (2 - $30 \mathrm{mg} / \mathrm{L})$ devido a solubilização de material orgânico da liteira (Thurman, 1985), logo a dinâmica da água subsuperficial e consequente saturação do perfil, atrelada a lixiviação do DOC estocado no solo irá promover o incremento na concentração de carbono orgânico no canal (Hinton et al., 1998; McGlynn \& McDonnell, 2003a; Lambert et al., 2011; Lambert et al., 2013). A relação entre a extensão das áreas saturadas e a produção e transporte de DOC foi modelada em Birkel et al. (2014) e Dick et al. (2015), considerando que a extensão dinâmica das áreas saturadas associada a decomposição de material orgânico em solos faz com que existam áreas fonte de CO nas governando a produção, armazenamento e transferência de DOC em direção aos canais.

Assim, a expansão e contração das áreas variáveis de afluência em resposta a períodos de seca e incrementos de precipitação modificam de forma significativa a dinâmica do escoamento subsuperficial, afetam a capacidade de decomposição e mineralização da matéria orgânica e consequente concentração de DOC no canal (Inamdar \& Mitchell, 2006; Tiwari et al., 2014). Em ambientes florestais em regiões temperadas (Fiebig et al., 1990) e subtropicais (Sorribas et al., 2012), durante eventos pluviométricos as fontes primárias de DOC são 
alóctones e relacionados a disponibilidade de matéria orgânica superficial (Hope et al., 1997; Hinton et al., 1998; Hood et al., 2006; Lambert et al., 2011; Lambert et al., 2013). Para a escala da bacia hidrográfica, a integração do comportamento hidrológico, relacionados a transferência de DOC, e a própria variação sazonal de DOC em solos tornam-se essencial para descrever as relações entre armazenamento e transferência de carbono para os canais (Dawson et al., 2008; Eimers et al., 2008).

De maneira geral, a Figura 3 apresenta um modelo conceitual do funcionamento de uma bacia hidrográfica de cabeceira, enfatizando a produção, armazenamento e transferência das frações de carbono orgânico entre encostas, zonas ripárias e canal. Assume-se, de maneira geral, que a mobilização de DOC é potencializada nas áreas saturadas em virtude da ascensão do nível freático (n.a.) em contato com horizontes superiores do solo enriquecidos com matéria orgânica, relacionado com a dinâmica espacial e temporal das áreas saturadas e consequente aporte no canal (QSub). Além disso, observa-se a contribuição de POC e DOC ao canal via processos de escoamento superficial (QSup). Desta maneira, em virtude da natureza histerética e mediada por thresholds, assume-se que as contribuições de água (Q) e CO nos canais assumem um comportamento não linear em função da disponibilidade de frações de carbono orgânico na paisagem possíveis de serem mobilizadas em paralelo ao ciclo hidrológico e sedimentológico na paisagem.

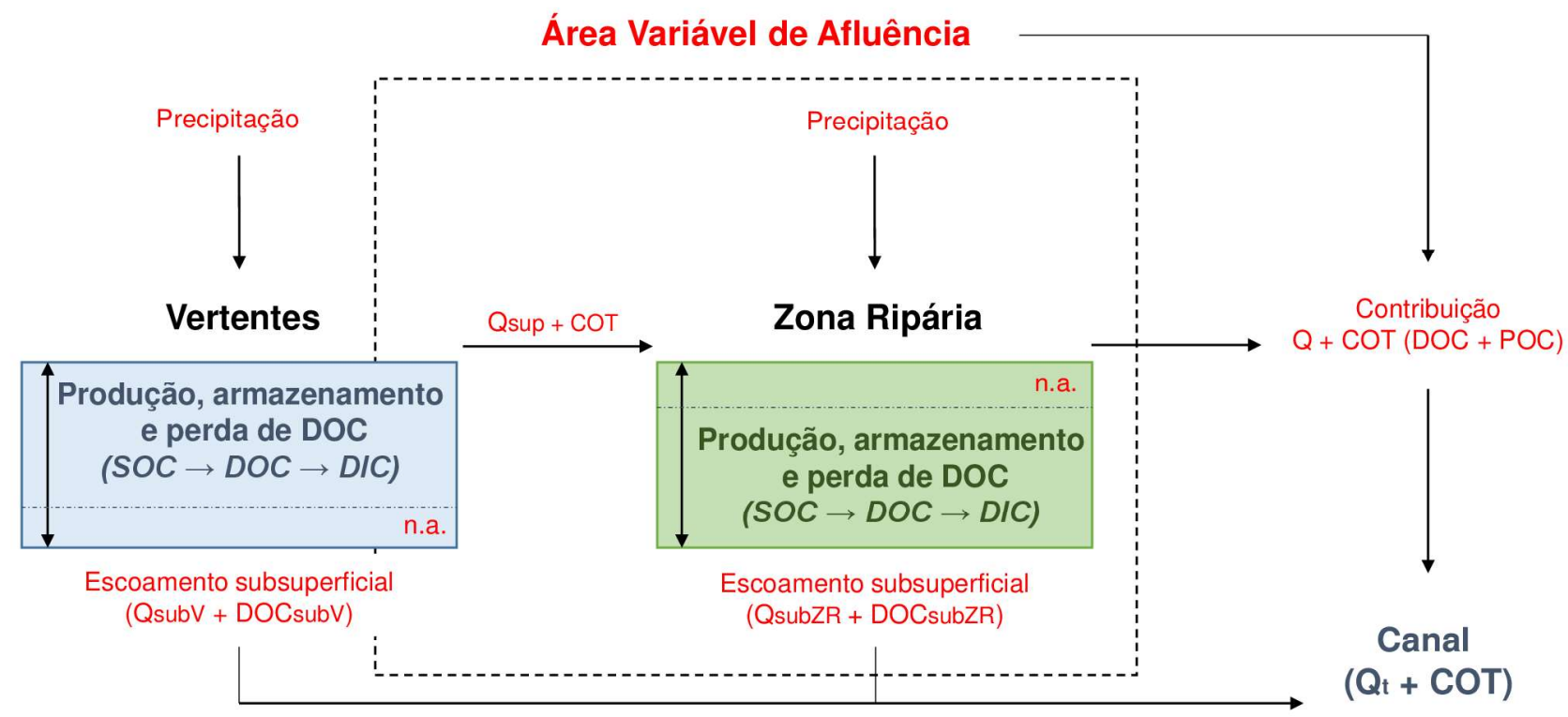

Figura 3 - Modelo conceitual da interação entre vertentes, zonas ripárias e canal no ciclo do carbono orgânico em bacias hidrográficas.

Morel et al. (2009), a partir da separação do hidrograma em 4 componentes (precipitação, aquífero profundo, água no perfil do solo e água estocada nas vertentes) estimam que apenas os dois últimos componentes contribuem de forma significativa para a variação de DOC no canal. Neste sentido, o escoamento de base atua como um continuum hidrológico e biogeoquímico permanente entre a vertente e o canal, enquanto que o escoamento superficial propicia um rápido incremento de carbono orgânico em direção aos canais (Fiebig et al., 1990), sobretudo em eventos de grande magnitude, capazes de superar os thresholds superficiais para geração de escoamento. McGlynn \&
McDonnell (2003b) e Sanderman et al. (2009) concluem que o escoamento subsuperficial é o maior contribuinte de DOC nos canais, considerando que as maiores concentrações ocorrem na ascensão do hidrograma em virtude da disponibilidade e facilidade de transporte das frações lábeis de carbono no solo durante eventos pluviométricos.

Diversos estudos evidenciam que as perdas de nutrientes e carbono ocorrem em eventos pluviométricos (Boyer et al., 1996; McGlynn \& McDonnell,2003a; Wagner et al., 2008; Lambert et al., 2011) e como os caminhos preferenciais interferem nas alterações biogeoquímicas do canal durante os eventos (Wagner 
et al., 2008; Temnerud et al., 2010), entretanto diversas incertezas e lacunas na descrição dos mecanismos de armazenamento e transferência do carbono orgânico dissolvido dos solos aos canais (Wagner et al., 2008; Mei et al., 2012). Por exemplo, devido ao padrão histerético da resposta da variação da concentração de DOC no canal observa-se a ocorrência do pico de DOC antes (Boyer et al., 1996) ou depois do pico de vazão na bacia hidrográfica (Hangen et al., 2001). Desta maneira, a diferença entre os picos máximos de vazão e da concentração de DOC no canal sugere que os mecanismos que controlam o armazenamento e a transferência de DOC aos canais são espacial e temporalmente dinâmicos (Wagner et al., 2008).

Conceitualmente, o reservatório de DOC no solo é reestabelecido nos períodos de recessão das vazões entre eventos (i.e. pela lixiviação de compostos orgânicos da camada superficial do solo e pela atividade microbiológica), sendo que a posterior dinâmica do aquífero raso impulsionada pela ocorrência de eventos pluviométricos promoverá a lixiviação destes compostos via escoamento subsuperficial, com acréscimo da concentração de DOC no sentido Vertente - Zona ripária, e posterior contribuição ao canal (McGlynn \& McDonnell, 2003a). Diversos estudos afirmam ainda que a saturação prolongada do perfil do solo, sobretudo em zonas ripárias, atua como fonte primária de DOC em bacias hidrográficas (Dosskey \& Bertsch, 1994; Hinton et al., 1998; McGlynn \& McDonnell, 2003a; Laudon et al., 2004; Morel et al., 2009; Lambert et al., 2011; Lambert et al., 2013), devido a liberação e desagregação de DOC dos agregados do solo, sobretudo em função da dissolução dos óxidos de ferro.

Por outro lado, nos horizontes subsuperficiais do solo, especialmente nos que contém baixa concentração de carbono orgânico e altas concentrações de alumínio e ferro, observa-se um potencial de adsorção de DOC considerando a movimentação horizontal de água pelo solo, resultando em baixas concentrações de DOC. Assim, afirma-se que subsuperficialmente não existe uma sazonalidade definida na concentração de DOC no solo, ao contrário da variação temporal da concentração em horizontes superficiais (Liu \& Sheu, 2003), cuja sazonalidade é definida pela disponibilidade de matéria orgânica, temperatura e o grau de umidade do solo.

A relação entre a disponibilidade de DOC na água residual do solo e a temperatura do perfil é diretamente proporcional, uma vez que a taxa de decomposição/ oxidação do carbono orgânico é reconhecida por ser limitada por baixas temperaturas e pela umidade do solo (Kalbitz et al., 2000), sendo potencializada em períodos secos e quentes (Billet et al., 2006). O aumento na temperatura dos horizontes superficiais do solo pode ser identificado como um fator que contribui para o incremento de liberação de DOC devido à ação catalizadora nos processos de decomposição e mineralização de matéria orgânica (Evans et al., 2006; Dawson \& Smith, 2007; Winterdahl et al., 2011), potencializando a mobilização de carbono orgânico (Dawson et al., 2008).

Integrar a relação entre o volume de escoamento e concentrações químicas nos solos das zonas ripárias pode ser utilizado para estimar a variabilidade química da água nos canais (Bishop et al., 2004). O movimento subsuperficial, em escala temporal de horas a dias, faz com que a água adquira as características químicas do perfil do solo. Assim, esse enriquecimento químico da água do solo, fará com que o escoamento subsuperficial tenha características químicas das vertentes e, sobretudo, das zonas ripárias, uma vez que os solos ripários são os últimos a terem contato com a água antes do aporte no canal (Bishop et al., 2004). Com um resultado ligeiramente diferente ao visar compreender a variação da concentração e fluxos de DOC no canal a partir da separação de hidrograma em 3 componentes (escoamento superficial, água no solo + escoamento subsuperficial e aquífero profundo) em uma vertente sem a presença de zona ripária, Chaplot \& Ribolzi (2014) indicam que ocorre a decomposição e estabilização da matéria orgânica transportada via DOC ao longo da encosta. Esta perda de CO ao longo da encosta via emissão para atmosfera ou estabilização no solo corresponderia a uma taxa de aproximadamente $80 \%$ da concentração de DOC obtido no terço superior da encosta em relação ao terço inferior. Desta maneira, estima-se que parte do DOC mobilizado durante eventos fica armazenado na vertente, em virtude da desconectividade temporária com o canal.

Diversos estudos identificaram os processos hidrológicos originários na zona ripária como mecanismos de controle da variabilidade biogeoquímica nos canais em bacias florestadas (Fiebig et al., 1990; Dosskey \& Bertsch, 1994; Hinton et al., 1998; McDonnell et al., 1998; Bishop et al., 2004; Lambert et al., 2011). Logo, as características químicas da zona ripária promovem uma assinatura química na água em 
função das características distintas do perfil de solos a montante do local de amostragem, por exemplo, o incremento nas concentrações de $\mathrm{Ca}, \mathrm{DOC}$ e $\mathrm{Cl}$ da água escoada subsuperficialmente a partir das condições de mistura com a água residual do perfil do solo (old water) (Bishop et al, 1995, Bishop et al., 2004).

Em função disso, a concentração de DOC em diversas fontes e no canal pode ainda ser utilizada como elemento traçador para separação dos componentes do hidrograma (Brown et al., 1999; McGlynn \& McDonnell, 2003b; Casper et al., 2003; Carey \& Quinton, 2005; Hood et al., 2006; Trček et al., 2006; Inamdar \& Mitchell, 2006; James \& Roulet, 2009; Chaplot \& Ribolzi, 2014) devido a possibilidade de caracterização da qualidade (Hood et al., 2006) e concentração do elemento traçador. Considerando que o tempo de transferência da água da encosta e solo durante eventos raramente excede algumas horas, a concentração de DOC pode ser considerada como um soluto com caráter quasi-conservativo até o aporte no canal (Casper et al., 2003). Assim, a caracterização do comportamento e contribuição de DOC no canal pode auxiliar a determinação não somente das áreas fontes, mas também de características químicas associadas a própria ciclagem do carbono orgânico (Hood et al., 2006), como por exemplo, processos químicos de redução de ferro no perfil do solo (Lambert et al., 2013).

\section{CONSIDERAÇÕES FINAIS}

A variabilidade temporal da concentração de carbono orgânico no canal e demais reservatórios da bacia hidrográfica relaciona-se com a ocorrência de processos biogeoquímicos associados a um comportamento hidrológico não-linear das unidades de paisagem que compõe a própria bacia. Afirma-se que a heterogeneidade espacial da concentração de SOC e produção de DOC na paisagem é um dos mecanismos que controlam a concentração de carbono orgânico na rede de drenagem. Desta maneira, evidenciam-se comportamentos distintos nas vertentes e zonas ripárias mediante inputs semelhantes, culminando em aportes não-lineares de carbono orgânico no canal durante e entre eventos pluviométricos. Assim, reconhecem-se diversas incertezas na descrição da dinâmica de carbono orgânico em bacias hidrográficas de cabeceira.

Em linhas gerais, indica-se a existência de controle das condições de umidade antecedente, nível do aquífero raso e temperatura no solo para produção, armazenamento e transferência de DOC nas vertentes e na zona ripária enquanto que a transferência de POC em direção aos canais relaciona-se com a quantidade $\mathrm{e}$ disponibilidade de material desagregado e magnitude dos eventos que desencadearão processos erosivos permitindo o seu transporte até a rede de drenagem. Entretanto, algumas questões importantes ainda permanecem em aberto, sobretudo as relacionadas as formas de enriquecimento de carbono na água e sedimentos ao longo das vertentes, transporte e deposição de frações orgânicas de carbono nas zonas ripárias e canais durante e entre eventos pluviométricos e processos biogeoquímicos associados.

\section{REFERÊNCIAS BIBLIOGRÁFICAS}

AITKENHEAD, J.A.; HOPE, D.; BILLETT, M.F. The relationship between dissolved organic carbon in streamwater and soil organic carbon pools at different spatial scales. Hydrological Processes. v.13, p.1289-1302, 1999.

BALDOCK, J.A.; SKJEMSTAD, J.O. Role of the soil matrix and minerals in protecting natural organic materials against biological attack. Organic Geochemistry, v. 31, n. 7, p. 697$710,2000$.

BILLETT, M.F.; DEACON, C.M.; PALMER, S.M.; DAWSON, J.J.C.; HOPE, D. Connecting organic carbon in streamwater and soils in a peatland catchment. Journal of Geophysical Research: Biogeosciences (2005-2012), v. 111, n. G2, 2006.

BIRKEL, C., SOULSBY, C., TETZLAFF, D. Integrating parsimonious models of hydrological connectivity and soil biogeochemistry to simulate stream DOC dynamics. Journal of Geophysical Research: Biogeosciences, 2014.

BISHOP, K.H.; LEE, Y.H.; PETTERSSON, C.; ALLARD, B. Terrestrial sources of methylmercury in surface waters: the importance of the riparian zone on the Svartberget catchment. Water, Air and Soil Pollution, v.80, p.435-444, 1995.

BISHOP, K.; SEIBERT, J.; KÖHLER, S.; LAUDON, H. Resolving the double paradox of rapidly mobilized old water with highly variable responses in runoff chemistry. Hydrological Processes, v.18, n.1, p.185-189, 2004.

BOYER, E.W.; HORNBERGER, G.M.; BENCALA, K.E.; McKNIGHT, D. Overview of a simple model describing variation of dissolved organic carbon in an upland watershed. Ecological Modelling, v.86, p.183-188, 1996. 
BROWN, V.A.; McDONNELL, J.J.; BURNS, D.A.; KENDALL, C. The role of event water, a rapid shallow flow component, and catchment size in summer stormflow. Journal of Hydrology, v.217, n³-4, p.171-190, 1999.

CAREY, S.K.; QUINTON, W.L. Evaluating runoff generation during summer using hydrometric, stable isotope and hydrochemical methods in a discontinuous permafrost alpine catchment. Hydrological Processes, v.19, n¹, p.95-114, 2005.

CASPER, M.C.; VOLKMANN, H.N.; WALDENMEYER, G.; PLATE, E.J. The separation of flow pathways in a sandstone catchment of the Northern Black Forest using DOC and a nested approach. Physics and Chemistry of the Earth, Parts $A / B / C$, v.28, n.6, p. 269-275, 2003.

CHAPLOT, V.A.M.; RUMPEL, C.; VALENTIN, C. Water erosion impact on soil and carbon redistributions within uplands of Mekong River. Global biogeochemical cycles, v. 19, n. 4, 2005.

CHAPLOT, V.; RIBOLZI, O. Hydrograph separation to improve understanding of Dissolved Organic Carbon Dynamics in Headwater catchments. Hydrological Processes, 28(21), 53545366. 2014.

CLARK, J.M.; LANE, S.N.; CHAPMAN, P.J.; ADAMSON, J.K. Export of dissolved organic carbon from an upland peatland during storm events: implications for flux estimates. Journal of Hydrology, 347(3), 438-447, 2007.

COLE, J.J.; PRAIRIE, Y.T.; CARACO, N.F.; McDOWELL, W.H.; TRANVIK, L.J.; STRIEGL, R.G.; DUARTE, C.M.; KORTELAINEN, P.; DOWNING, J.A.; MIDDLELBURG, J.J.; MELACJ, J. Plumbing the global carbon cycle: integrating inland waters into the terrestrial carbon budget. Ecosystems, v. 10, n. 1, p. 172-185, 2007.

DALZELL, B.J.; FILLEY, T.R.; HARBOR, J.M. The role of hydrology in annual organic carbon loads and terrestrial organic matter export from a midwestern agricultural watershed. Geochimica et Cosmochimica Acta, v. 71, n. 6, p. 1448-1462, 2007.

DAVIDSON, E.A.; JANSSENS, I.A. Temperature sensitivity of soil carbon decomposition and feedbacks to climate change. Nature, v. 440, n.7081, p. 165-173, 2006.

DAWSON, J.J.; BILLET, M.F.; HOPE, D.; PALMER, S.M.; DEACON, C.M. Sources and sinks of aquatic carbon in a peatland stream continuum. Biogeochemistry, v. $70, \mathrm{n}^{\circ} 1$, p. 71 92, 2004.

DAWSON, J.J.C.; SMITH, P. Carbon losses from soil and its consequences for land-use management. Science of the Total Environment, v. 382, n. 2, p. 165-190, 2007.

DAWSON, J.J.C.; SOULSBY, C.; TETZLAFF, D.; HRACHOWITZ, M.; DUNN, S.M.; MALCOLM, I.A. Influence of hydrology and seasonality on DOC exports from three contrasting upland catchments. Biogeochemistry, v.90, ${ }^{\circ} 1$, p.93-113, 2008.

DICK, J. J., TETZLAFF, D., BIRKEL, C., SOULSBY, C. Modelling landscape controls on dissolved organic carbon sources and fluxes to streams. Biogeochemistry, 122(2-3), 361-374, 2015.

DORAN, J.W.; PARKIN, T.B. Defining and assessing soil quality. SSSA special publication, 35, 3-3, 1994.

DOSSKEY, M.G.; BERTSCH, P.M. Forest sources and pathways of organic matter transport to a blackwater stream: a hydrologic approach. Biogeochemistry, v. 24, n. 1, p. 1-19, 1994.

EGLIN, T., WALTER, C., NYS, C., FOLLAIN, S., FORGEARD, F., LEGOUT, A., SQUIVIDANT, H. Influence of waterlogging on carbon stock variability at hillslope scale in a beech forest (Fougères forest-West France). Annals of forest science, 65(2), 1-10, 2008.

EIMERS, M.C.; WATMOUGH, S.A.; BUTTLE, J.M. Long-term trends in dissolved organic carbon concentration: a cautionary note. Biogeochemistry. v.87, p.71-81, 2008.

EVANS, C.D.; CHAPMAN, P.J.; CLARK, J.M.; MONTEITH, D.T.; CRESSER, M.S. Alternative explanations for rising dissolved organic carbon export from organic soils. Global Change Biology. v.12, p.2044-2053, 2006.

FALKOWSKI, P., SCHOLES, R. J., BOYLE, E. E. A., CANADELL, J., CANFIELD, D., ELSER, J., GRUBER, N.; HIBBARD, K.; HOGBERG, P.; LINDER, S.; MACKENZIE, F.T.; MOORE III, B.; PEDERSEN, T.; ROSENTHAL, Y.; SEITZINGER, S.; SMETACEK, V.; Steffen, W. The global carbon cycle: a test of our knowledge of earth as a system. Science, 290(5490), 291-296, 2000.

FIEBIG, D.M.; LOCK, M.A.; NEAL, C. Soil water in the riparian zone as a source of carbon for a headwater stream. Journal of Hydrology, v.116, n.1, p.217-237, 1990.

FRANK, H.; PATRICK, S.; PETER, W.; HANNES, F. Export of dissolved organic carbon and nitrogen from Gleysol dominated catchments-the significance of water flow paths. Biogeochemistry, v.50, $\mathrm{n}^{\circ} .2$, p.137-161, 2000.

GENEVIÈVE, A.; BIRKEL, C.; TETZLAFF, D.; SOULSBY, C.; McDONNELL, J.J.; TAROLLI, P. A comparison of wetness 
indices for the prediction of observed connected saturated areas under contrasting conditions. Earth Surface Processes and Landforms, 39(3), 399-413, 2014.

HANGEN, E.; LINDENLAUB, M., LEIBUNDGUT, C.; von WILPERT, K. Investigating mechanisms of stormflow generation by natural tracers and hydrometric data: a small catchment study in the Black Forest, Germany. Hydrological Processes 15: 183-199, 2001.

HINTON, M.J.; SCHIFF, S.L.; ENGLISH, M.C. Sources and flowpaths of dissolved organic carbon during storms in two forested watersheds of the Precambrian Shield. Biogeochemistry, v.4, p.175-197, 1998.

HONGVE, D. Production of dissolved organic carbon in forested catchments. Journal of Hydrology, v. 224, n. 3, p. 91-99, 1999.

HOOD, E.; GOOSEFF, M.N.; JOHNSON, S.L. Changes in the character of stream water dissolved organic carbon during flushing in three small watersheds, Oregon. Journal of Geophysical Research: Biogeosciences (2005-2012), v.111, n.G1, 2006.

HOFFMANN, T.; GLATZEL, S.; DIKAU, R. A carbon storage perspective on alluvial sediment storage in the Rhine catchment. Geomorphology, v. 108, n. 1, p. 127-137, 2009.

HOPE, D.; BILLETT, M.F.; MILNE, R.; BROWN, T.A. Exports of organic carbon in British rivers. Hydrological Processes, v.11, n³, p.325-344, 1997.

HOPE, D., PALMER, S.M., BILLETT, M.F.; DAWSON, J.J.C. Variations in dissolved $\mathrm{CO} 2$ and $\mathrm{CH} 4$ in a first order stream and catchment: An investigation of soil-stream linkages, Hydrological Processes, 18, 3255- 3275, 2004.

INAMDAR, S.P.; MITCHELL, M.J. Hydrologic and topographic controls on storm-event exports of dissolved organic carbon (DOC) and nitrate across catchment scales. Water Resources Research, 42(3), 2006.

JAMES, A.L., ROULET, N.T. Antecedent moisture conditions and catchment morphology as controls on spatial patterns of runoff generation in small forest catchments. Journal of Hydrology. 377 (3-4), 351-366, 2009.

JOBBÁGY, E.G.; JACKSON, R.B. The vertical distribution of soil organic carbon and its relation to climate and vegetation. Ecological Applications, 10:423-436, 2000

KALBITZ, K.; SOLINGER, S.; PARK, J.H.; MICHALZIK, B.; MATZNER, E. Controls on the dynamics of dissolved organic matter in soils: a review, Soil Science, v.165, p.277-304, 2000.
KNAPIK, H.G. Organic Matter Characterization and Modeling in Polluted Rivers for Water Quality Planning and Management. Curitiba: UFPR/DHS, Tese (Doutorado em Engenharia de Recursos Hídricos e Ambiental) - Pós-Graduação em Engenharia de Recursos Hídricos e Ambiental - PPGERHA, Universidade Federal do Paraná, 318p., 2014.

LAFLEUR, P.M., ROULET, N.T., BUBIER, J.L, FROLKING, S. MOORE, T.R. Interannual variability in the peatland-atmosphere carbon dioxide exchange at an ombrotrophic bog, Global Biogeochemical Cycles, 17(2), 1036, 2003.

LAL, R. Global soil erosion by water and carbon dynamics. In: LAL, R. Soils Processes and the Carbon Cycle, 1995.

LAL, R. Soil carbon sequestration to mitigate climate change. Geoderma. vol. 123, p.1-22, 2004.

LAL, R. Forest soils and carbon sequestration. Forest Ecology and Management, v.220, p.242-258, 2005.

LAMBERT, T.; PIERSON-WICKMANN, A.C.; GRUAU, G.; THIBAULT, J.N.; JAFFREZIC, A. Carbon isotopes as tracers of dissolved organic carbon sources and water pathways in headwater catchments. Journal of Hydrology, v. 402, n. 3, p. 228-238, 2011.

LAMBERT, T.; PIERSON-WICKMANN, A.C.; GRUAU, G.; JAFFREZIC, A.; PETITJEAN, P.; THIBAULT., J.N.; JEANNEAU, L. Hydrologically driven seasonal changes in the sources and production mechanisms of dissolved organic carbon in a small lowland catchment. Water Resources Research, v. 49, n. 9, p. 5792-5803, 2013.

LAUDON, H.; BERGGREN, M.; ÅGREN, A.; BUFFAM, I.; BISHOP, K.; GRABS, T.; JANSSON, M.; KOHLER, S. Patterns and dynamics of dissolved organic carbon (DOC) in boreal streams: the role of processes, connectivity, and scaling. Ecosystems, 14(6), 880-893, 2011.

LAUDON, H.; KÖHLER, S.; BUFFAM, I. Seasonal TOC export from seven boreal catchments in northern Sweden. Aquatic Sciences, v. 66, n. 2, p. 223-230, 2004.

LENNON, J.T.; Experimental evidence that terrestrial carbon subsidies increase $\mathrm{CO} 2$ flux from lake ecosystems, Oecologia, 138, 584-591, 2004.

LIU, C.P.; SHEU, B.H. Dissolved organic carbon in precipitation, throughfall, stemflow, soil solution, and stream water at the Guandaushi subtropical forest in Taiwan. Forest Ecology and Management, v.172, n.2, p.315-325, 2003.

MARQUES, J.D.O; LUIZAO, F.J.; TEIXEIRA, W.G.; FERREIRA, S.J. F. Variações do carbono orgânico dissolvido 
e de atributos físicos do solo sob diferentes sistemas de uso da terra na Amazônia central. Revista Brasileira de Ciências do Solo. vol.36, n.2, p.611-622, 2012.

McDONNELL, J.J.; McGLYNN, B.L.; KENDALL, K.; SHANLEY, J.; KENDALL, C. The role of near-stream riparian zones in the hydrology of steep upland catchments. IAHS Publications-Series of Proceedings and Reports-Intern Assoc Hydrological Sciences, v. 248, p. 173-180, 1998.

McGLYNN, B.L.; McDONNELL, J.J. Role of discrete landscape units in controlling catchment dissolved organic carbon dynamics. Water Resources Research, v. 39, n.. 4, p. 1090, 2003a.

McGLYNN, B.L.; McDONNELL, J.J. Quantifying the relative contributions of riparian and hillslope zones to catchment runoff. Water Resources Research, v. 39, n . 11, p. 1310, 2003 b.

MEI, Y.; HORNBERGER, G.M.; KAPLAN, L.A.; NEWBOLD, J.D.; AUFDENKAMPE, A.K. Estimation of dissolved organic carbon contribution from hillslope soils to a headwater stream. Water Resources Research, 48(9), 2012.

MEYBECK, M. Carbon, nitrogen, and phosphorus transport by world rivers. American Journal of Science, v.282: p. 401-450, 1982.

MOREL, B.; DURAND, P.; JADDREZIC, A.; GRUAU, G.; MOLENAT, J. Sources of dissolved organic carbon during stormflow in a headwater agricultural catchment. Hydrological Processes, v. 23, n. 20, p. 2888-2901, 2009.

RITCHIE, J.C.; McCARTY, G.W.; VENTERIS, E.R.; KASPER, T.C. Soil and soil organic carbon redistribution on the landscape. Geomorphology, v.89, nº 1, p.163-171, 2007.

SANDERMAN, J.; LOHSE, K.A.; BALDOCK, J.A.; AMUNDSON, R. Linking soils and streams: Sources and chemistry of dissolved organic matter in a small coastal watershed, Water Resources Research, 45, 2009.

SCHMIDT MWI, TORN MS, ABIVEN S, DITTMAR T, GUGGENBERGER G, JANSSENS IA, KLEBER M, KÖGELKNABNER I, MANNING DAC, NANNIPIERI P, RASSE DP,

WEINER S, TRUMBORE SE. Persistence of soil organic matter as an ecosystem property. Nature 478: 49-56, 2011.

SCHWANGHART, W.; JARMER, T. Linking spatial patterns of soil organic carbon to topography - a case study from southeastern Spain. Geomorphology, v. 126, n. 1, p. 252-263, 2011.

SCOTT, M.J.; JONES, M.N.; WOOF, C.; TIPPING, E. Concentrations and fluxes of dissolved organic carbon in drainage water from an upland peat system. Environment International, v.24, n.5, p.537-546, 1998.

SIEFERT, C.A.C. Dinâmica do aquífero raso e fontes de carbono orgânico dissolvido em uma bacia hidrográfica de cabeceira. Curitiba: UFPR/PPGG, Tese (Doutorado em Geografia) - Programa de Pós-Graduação em Geografia, Universidade Federal do Paraná, 115p., 2016.

SORRIBAS, M.V. Simulação da dinâmica de carbono em bacias hidrográficas. Dissertação (Instituto de Pesquisas Hidráulicas, Programa de Pós-graduação em Recursos Hídricos e Saneamento Ambiental, Universidade Federal do Rio Grande do Sul). Porto Alegre: UFRGS, 141p., 2011.

SORRIBAS, M. V.; COLLISCHONN, W.; MARQUES, D.M.M.; FRAGOSO JÚNIOR, C.R.; CASTRO, N.M.R.; SOUZA, R.S. Modelagem Distribuída do Carbono em Bacias Hidrográficas. Revista Brasileira de Recursos Hídricos, v. 17, p. 225-240, 2012.

STARR, G.C.; LAL, R.; KIMBLE, J. Empirical relationships for soil organic carbon transport from agricultural watersheds in Ohio. Land Degradation \& Development, v. 19, n. 1, p. 57-64, 2008.

STIEGLITZ, M.; SHAMAN, J.; McNAMARA, J.; ENGEL, V.; SHANLEY, J.; KLING, G.W. An approach to understanding hydrologic connectivity on the hillslope and the implications for nutrient transport. Global Biogeochemical Cycles, v. 17(4), 2003.

SWARZENSKI, P.; CAMPBELL, P.L. On the Worldwide Riverine Transport of Sediment-Associated Contaminants to the Ocean. In.: ANDERSON, M.; McDONNELL., J.J. Encyclopedia of Hydrological Sciences. John Wiley \& Sons Ltd. 2005.

TEMNERUD, J.; SEIBERT, J.; JANSSON, M.; BISHOP, K. Spatial variation in discharge and concentrations of organic carbon in a catchment network of boreal streams in northern Sweden. Journal of Hydrology, v. 342, n. 1, p. 72-87, 2007.

TEMNERUD, J.; FÖLSTER, J., BUFFAM, I.; LAUDON, H.; ERLANDSSON, M.; BISHOP, K. Can the distribution of headwater stream chemistry be predicted from downstream observations? Hydrological Processes, v.24, n 16 , p.22692276, 2010.

THOMAZ, E.L.; VESTENA, L.R. Measurement of runoff and soil loss from two differently sized plots in a subtropical environment (Brazil). Earth Surface Processes and Landforms, v.37, p.363-373, 2012. 
THURMAN, E.M. Organic Geochemistry of Natural Waters. Developments in Biogeochemistry, v.2, 1985.

TISDALL, J. M.; OADES, J_M. Organic matter and waterstable aggregates in soils. Journal of Soil Science, v. 33, n. 2, p. 141-163, 1982.

TIWARI, T.; LAUDON, H.; BEVEN, K.; ÅGREN, A.M. Downstream changes in DOC: Inferring contributions in the face of model uncertainties. Water Resources Research, 50(1), 514-525, 2014.

TRČEK, B.; VESELIČ, M.; PEZDIČ, J. The vulnerability of karst springs - a case study of the Hubelj spring (SW Slovenia). Environmental Geology, v.49, n.6, p. 865-874, 2006.

VAN VERSEVELD, W. J., McDONNELL, J. J., LAJTHA, K. A mechanistic assessment of nutrient flushing at the catchment scale. Journal of Hydrology, 358(3), 268-287, 2008.

VAN VERSEVELD, W.J.; McDONNELL, J.J.; LAJTHA, K. The role of hillslope hydrology in controlling nutrient loss. Journal of Hydrology, v.367, n³, 177-187, 2009.

WAGNER, L.E.; VIDON, P.; TEDESCO, L.P.; GRAY, M. Stream nitrate and DOC dynamics during three spring storms across land uses in glaciated landscapes of the Midwest. Journal of Hydrology, 362(3), 177-190, 2008.

WATERLOO, M.J.; OLIVEIRA, S.M.; DRUCKER, D.P.; NOBRE, A.D.; CUARTAS, L.A.; HODNETT, M.G.; LANGEDIJK, I.; JANS, W.W.P.; TOMASELLA, J.; ARAUJO, A.C. PIMENTEL., T.P.; ESTRADA, J.C.M. Export of organic carbon in run-off from an Amazonian rainforest blackwater catchment. Hydrological Processes, v. 20, n. 12, p. 2581-2597, 2006.

WINTERDAHL, M.; TEMNERUD, J.; FUTTER, M.N.; LÖGFREN, S.; MOLDAN, F.; BISHOP, K. Riparian zone influence on stream water dissolved organic carbon concentrations at the Swedish Integrated Monitoring sites. Ambio, v.40, n.8, p.920-930, 2011.

YOO, K.; AMUNDSON, R.; HEIMSATH, A.M.; DIETRICH, W.E. Spatial patterns of soil organic carbon on hillslopes: integrating geomorphic processes and the biological $\mathrm{C}$ cycle. Geoderma. v.130, p.47-65, 2006.

ZUCCO, E.; PINHEIRO, A.; SOARES, P.A.; Concentrações de nutrientes e de carbono transportados por ondas de cheia em uma bacia agrícola no estado de Santa Catarina. Revista Brasileira de Recursos Hídricos, v. 20, p. 369-378, n. 2015. 